\title{
Complete axiomatization of a relative modal logic with composition and intersection
}

\author{
Philippe Balbiani, Luis Fariñas del Cerro \\ Laboratoire d'informatique de Paris-Nord \\ Institut de recherche en informatique de Toulouse
}

\begin{abstract}
We consider the question of the complete axiomatization of a relative modal logic with composition and intersection.

\section{Introduction}

As an abstraction of a system introduced by Pratt, propositional dynamic logic $-P D L-$ is a powerful instrument with which it is possible to reason about programs $[9,6,7,11,8]$. The linguistic basis of $P D L$ is the propositional calculus enlarged with a family of modal operators of the form $[\alpha]$ where $\alpha$ is a program in $\Pi_{c}$ composed inductively via regular rules in the following way, for every $\alpha, \beta \in \Pi_{c}: \alpha ; \beta$ $\in \Pi_{c}$ — "do $\alpha$ followed by $\beta$ ", $\alpha \vee \beta \in \Pi_{c}$ — "do either $\alpha$ or $\beta ", \alpha^{+} \in$ $\Pi_{c}$ - "repeat $\alpha$ a finite number of times", the meaning of the formula $[\alpha] A$ being that "after every terminating execution of $\alpha$, it is the case that $A$ ". Semantically, the program $\alpha$ in $\Pi_{c}$ corresponds to the binary relation $R(\alpha)$ between the states of some presumed universe $W$, seeing that $R(\alpha ; \beta)=R(\alpha) \circ R(\beta), R(\alpha \vee \beta)=R(\alpha) \cup R(\beta), R\left(\alpha^{+}\right)=$ $R(\alpha)^{+}$, the formula $[\alpha] A$ being true in the state $x \in W$ when $A$ is true in every state $y$ such that $x R(\alpha) y$. Since the days Gabbay, Parikh and Segerberg gave their proofs of the completeness of the axiomatization presented by Segerberg, several extensions of $P D L$ have been
\end{abstract}


proposed. One of them adds the intersection operator of programs, inductively allowing, for every $\alpha, \beta \in \Pi_{c}, \alpha \wedge \beta \in \Pi_{c}-$ "do $\alpha$ and $\beta$ in parallel", considering that $R(\alpha \wedge \beta)=R(\alpha) \cap R(\beta)$.

The interest to introduce the intersection operator of programs lies in the fact that it formalizes some aspects of what is known as parallelism. That is the reason why various authors have considered the following issues : decidability / complexity and axiomatization / completeness of a $P D L$ with intersection $[10,3,16]$. From the decidability / complexity point of view, Harel [10] proves that the validity problem for a $P D L$ with intersection, tests and deterministic atomic programs is undecidable. In the general case, non-deterministic atomic programs are allowed and Danecki [3] proves that the validity problem for a $P D L$ with intersection and tests is decidable. From the axiomatization / completeness point of view, Passy and Tinchev [16] enrich a $P D L$ with intersection, tests and names to be interpreted as true at exactly one state and prove the completeness of an axiomatization of the resultant logic of programs, leaving open the question of the completeness of an axiomatization of a name-free test-free $P D L$ with intersection.

Considering the question of the complete axiomatization of a $P D L$ with intersection, we have to face the fact that neither the transitive closure of one binary relation nor the intersection of two binary relations are modally definable. This problem leads to the presentday impossibility of carrying out the plan of the complete axiomatization of a $P D L$ with intersection. Consequently, we do not claim to be able to realize this plan and we modestly focus our attention on a fragment of $P D L$ with intersection. The linguistic basis of this fragment is the propositional calculus enlarged with a family of modal operators of the form $[\alpha]$ where $\alpha$ is a program in $\Pi_{c}$ composed in the following way, for every $\alpha, \beta \in \Pi_{c}: \alpha ; \beta \in \Pi_{c}, \alpha \wedge \beta \in \Pi_{c}$. Semantically, the program $\alpha$ in $\Pi_{c}$ still corresponds to the binary relation $R(\alpha)$ between the states of some universe $W$, seeing that $R(\alpha ; \beta)=R(\alpha) \circ R(\beta)$, $R(\alpha \wedge \beta)=R(\alpha) \cap R(\beta)$.

The axiomatization of this fragment of $P D L$ with intersection is presented in the section 4 whereas the proof of the completeness of this axiomatization is organized in the sections 6 and 7 . The section 6 shows that if a formula $A$ is consistent with the considered axiomatical presentation of our relative modal logic with composition and intersection then there exists a universe $W$ and, for every $\alpha \in \Pi_{c}$, 
a binary relation $R(\alpha)$ between the states of $W$ such that $R(\alpha ; \beta) \supseteq$ $R(\alpha) \circ R(\beta), R(\alpha \wedge \beta)=R(\alpha) \cap R(\beta)$ and there exists a state $x \in W$ such that $A$ is true in $x$. However, it is quite possible that there exists $\alpha, \beta \in \Pi_{c}$ and there exists $x, z \in W$ such that $x R(\alpha ; \beta) z$ and, for every $y \in W$, either $x \overline{R(\alpha)} y$ or $y \overline{R(\beta)} z$ and the section 7 proves that the relational structure $(W, R)$ can be transformed into a relational structure $\left(W_{\omega}, R_{\omega}\right)$ such that $R_{\omega}(\alpha ; \beta)=R_{\omega}(\alpha) \circ R_{\omega}(\beta), R_{\omega}(\alpha \wedge \beta)$ $=R_{\omega}(\alpha) \cap R_{\omega}(\beta)$ and there exists a state $x \in W_{\omega}$ such that $A$ is true in $x$.

\section{Language}

Let $\Pi_{a}$ be a countable set of "atomic programs". The set $\Pi_{c}$ of the "complex programs" is defined by induction in the following way :

- $\alpha=\pi, \pi \in \Pi_{a}|\alpha ; \beta| \alpha \wedge \beta$.

Let $\succeq$ be the smallest ordering on $\Pi_{c}$ compatible with $\{\alpha ;(\beta ; \gamma)=$ $(\alpha ; \beta) ; \gamma\}$ and such that, for every $\alpha, \beta, \gamma \in \Pi_{c}$ :

- $\alpha \wedge \beta \succeq \alpha$.

- $\alpha \wedge \beta \succeq \beta$.

- If $\alpha \succeq \beta$ and $\alpha \succeq \gamma$ then $\alpha \succeq \beta \wedge \gamma$.

that is to say, if $\equiv$ is the binary relation on $\Pi_{c}$ defined by induction in the following way :

- $\alpha \equiv \beta$ iff there exists $K \geq 1$ such that $\alpha \equiv_{K} \beta$ where :

$-\alpha ;(\beta ; \gamma) \equiv_{1}(\alpha ; \beta) ; \gamma$ and $(\alpha ; \beta) ; \gamma \equiv_{1} \alpha ;(\beta ; \gamma)$.

$-\alpha \equiv_{1} \alpha$.

- If $\alpha \equiv_{K} \beta$ and $\beta \equiv_{L} \gamma$ the $\alpha \equiv_{K+L} \gamma$.

- If $\alpha \equiv_{K} \beta$ and $\gamma \equiv_{L} \delta$ then $\alpha ; \gamma \equiv_{K+L} \beta ; \delta$ and $\alpha \wedge \gamma \equiv_{K+L}$ $\beta \wedge \delta$.

then $\succeq$ is the binary relation on $\Pi_{c}$ defined by induction in the following way :

- $\alpha \succeq \beta$ iff there exists $K \geq 1$ such that $\alpha \succeq_{K} \beta$ where :

$-\alpha \wedge \beta \succeq_{1} \alpha$ and $\alpha \wedge \beta \succeq_{1} \beta$.

$-\alpha \succeq_{1} \alpha$.

- If $\alpha \succeq_{K} \beta$ and $\beta \succeq_{L} \gamma$ the $\alpha \succeq_{K+L} \gamma$. 
- If $\alpha \equiv \beta$ and $\beta \succeq_{L} \gamma$ the $\alpha \succeq_{K+1} \gamma$.

- If $\alpha \succeq_{K} \beta$ and $\beta \equiv \gamma$ the $\alpha \succeq_{K+1} \gamma$.

- If $\alpha \succeq_{K} \beta$ and $\gamma \succeq_{L} \delta$ then $\alpha ; \gamma \succeq_{K+L} \beta ; \delta$ and $\alpha \wedge \gamma \succeq_{K+L}$ $\beta \wedge \delta$.

- If $\alpha \succeq_{K} \beta$ and $\alpha \succeq_{L} \gamma$ then $\alpha \succeq_{K+L} \beta \wedge \gamma$.

\section{Semantical study}

Let $W$ be a nonempty set and $R$ be a mapping of $\Pi_{c}$ to $2^{W \times W}$. $(W, R)$ is called "frame". $(W, R)$ is "irreflexive" when, for every $\alpha \in \Pi_{c}$ :

- $R(\alpha) \cap I d_{W}=\emptyset$.

$(W, R)$ is "partial" when, for every $\alpha, \beta \in \Pi_{c}$ :

- $R(\alpha ; \beta)=R(\alpha) \circ R(\beta)$.

- If $\alpha \succeq \beta$ then $R(\alpha) \subseteq R(\beta)$.

$(W, R)$ is "standard" when, for every $\alpha, \beta \in \Pi_{c}$ :

- $R(\alpha ; \beta) \supseteq R(\alpha) \circ R(\beta)$.

- $R(\alpha \wedge \beta) \supseteq R(\alpha) \cap R(\beta)$.

- If $\alpha \succeq \beta$ then $R(\alpha) \subseteq R(\beta)$.

It should be remarked that :

Proposition 1 If $(W, R)$ is standard then, for every $\alpha, \beta \in \Pi_{c}, R(\alpha \wedge$ $\beta)=R(\alpha) \cap R(\beta)$.

$(W, R)$ is "normal" when, for every $\alpha, \beta \in \Pi_{c}$ :

- $R(\alpha ; \beta)=R(\alpha) \circ R(\beta)$.

- $R(\alpha \wedge \beta)=R(\alpha) \cap R(\beta)$.

It may be asserted that :

Proposition 2 If $(W, R)$ is normal then, for every $\alpha, \beta \in \Pi_{c}$ :

- If $\alpha \equiv \beta$ then $R(\alpha)=R(\beta)$.

- If $\alpha \succeq \beta$ then $R(\alpha) \subseteq R(\beta)$.

Consequently, the reader may easily verify that:

Proposition $3(W, R)$ is normal iff $(W, R)$ is partial and standard. 
Let $V$ be a mapping of $\Phi_{c}$ to $2^{W}$ such that, for every $A, B \in \Phi_{c}$ and for every $\alpha \in \Pi_{c}$ :

- $V(\neg A)=W \backslash V(A)$.

- $V(A \rightarrow B)=W \backslash V(A) \cup V(B)$.

- $V([\alpha] A)=\{x: R(\alpha)(x) \subseteq V(A)\}$.

$V$ is called "valuation on $(W, R)$ " and $(W, R, V)$ is called "model on $(W, R)$ defined from $V$ ". For every $A \in \Phi_{c}, A$ is "true in $(W, R, V)$ " when $V(A)=W$. Let $R A L$ be the set of the formulas true in every normal model. The main result of this paper is the proof of the completeness of the following axiomatization of $R A L$.

\section{Axiomatization}

Together with the classical tautologies, all the instances of the following schema, for every $\alpha \in \Pi_{c}$ :

- $[\alpha](A \rightarrow B) \rightarrow([\alpha] A \rightarrow[\alpha] B)$.

and all the instances of the following schemata, for every $\alpha, \beta \in \Pi_{c}$ :

- $[\alpha ; \beta] A \leftrightarrow[\alpha][\beta] A$.

- If $\alpha \succeq \beta$ then $[\alpha] A \leftarrow[\beta] A$.

are axioms of $R A L$. Together with the classical inference rules, all the instances of the following schema, for every $\alpha \in \Pi_{c}$ :

- If $A$ is a theorem then $[\alpha] A$ is a theorem.

are inference rules of $R A L$. The reader may easily verify that the axioms of $R A L$ are true in every partial model and the inference rules of $R A L$ preserve truth in every partial model. Consequently, a proof by induction on the length of the proof of $A$ will show that:

Theorem 1 For every $A \in \Phi_{c}$, if $A$ is a theorem of $R A L$ then $A$ is true in every partial model.

Let ? be a set of formulas :

- ? is "maximal" when, for every $A \in \Phi_{c}, A \in$ ? or $\neg A \in$ ?.

- ? is "consistent" when, for every $K \geq 1$ and for every $A_{1}, \ldots, A_{K}$ $\in ?, \neg\left(A_{1} \wedge \ldots \wedge A_{K}\right)$ is not a theorem of $R A L$. 
It may be asserted that :

Proposition 4 For every consistent set? of formulas, there exists a maximal and consistent set ?' of formulas such that? $\subseteq$ ?'.

Proposition 5 For every $\alpha \in \Pi_{c}$, for every $A \in \Phi_{c}$ and for every maximal and consistent set? of formulas, if $[\alpha] A \notin$ ? then there exists a maximal and consistent set $\Delta$ of formulas such that $[\alpha]$ ? $\subseteq \Delta$ and $A \notin \Delta$.

where $[\alpha] ?=\{A:[\alpha] A \in ?\}$.

Proposition 6 For every $\alpha, \beta \in \Pi_{c}$ and for every maximal and consistent set ?, $\Lambda$ of formulas, if $[\alpha ; \beta]$ ? $\subseteq \Lambda$ then there exists a maximal and consistent set $\Delta$ of formulas such that $[\alpha]$ ? $\subseteq \Delta$ and $[\beta] \Delta \subseteq \Lambda$.

Proof The proofs of the propositions 4, 5 and 6 use the techniques of the maximal and consistent sets of formulas [12].

\section{$5 \quad$ Functions of maximality}

To prove the completeness of the axiomatization of $R A L$, we use mappings called functions of maximality and structures called spaces of maximality. Let $(W, R)$ be a frame and $S$ be a mapping of $W$ to the set of the maximal and consistent sets of formulas such that, for every $x \in W$, for every $\alpha \in \Pi_{c}$ and for every $A \in \Phi_{c},[\alpha] A \in S(x)$ iff, for every $y \in W$, if $y \in R(\alpha)(x)$ then $A \in S(y)$. $S$ is called "function of maximality on $(W, R)$ " and $(W, R, S)$ is called "space of maximality on $(W, R)$ defined from $S$ ". For every $A \in \Phi_{c}, A$ is "true in $(W, R, S)$ " when, for every $x \in W, A \in S(x)$. The "valuation on $(W, R)$ associated to $S$ " is the valuation $V$ on $(W, R)$ such that, for every $P \in \Phi_{a}$, $V(P)=\{x: P \in S(x)\}$. A proof by induction on the complexity of $A$ will show that :

Proposition 7 For every $A \in \Phi_{c}, V(A)=\{x: A \in S(x)\}$.

From all this it follows that:

Proposition 8 For every $A \in \Phi_{c}, A$ is true in $(W, R, V)$ iff $A$ is true in $(W, R, S)$. 


\section{Proof of the standard completeness}

Let $W_{0}, W_{1}, \ldots$ be a sequence of sets defined by induction in the following way, $S$ be a mapping of $W=\bigcup\left\{W_{i}: i \geq 0\right\}$ to the set of the maximal and consistent sets of formulas such that :

- Let $W_{0}=\{0\}$ and $S(0)$ be a maximal and consistent set of formulas.

- For every $i \geq 1$, let $W_{i}=W_{i-1} \cup\left\{x \alpha A: x \in W_{i-1}, \alpha \in \Pi_{c}\right.$ and $A \in \Phi_{c}$ are such that $[\alpha] A \notin S(x)$ and $\left.x \alpha A \notin W_{i-1}\right\}$ and, according to the proposition 5 , for every $x \in W_{i-1}$, for every $\alpha$ $\in \Pi_{c}$ and for every $A \in \Phi_{c}$ such that $[\alpha] A \notin S(x)$ and $x \alpha A \notin$ $W_{i-1}, S(x \alpha A)$ be a maximal and consistent set of formulas such that $[\alpha] S(x) \subseteq S(x \alpha A)$ and $A \notin S(x \alpha A)$.

and $R$ be the mapping of $\Pi_{c}$ to $2^{W \times W}$ such that, for every $\alpha \in \Pi_{c}$ and for every $x, y \in W, x R(\alpha) y$ iff there exists $N \geq 1$, there exists $\alpha_{1}, \ldots, \alpha_{N} \in \Pi_{c}$ and there exists $A_{1}, \ldots, A_{N} \in \Phi_{c}$ such that $\bigcirc\left(\alpha_{1}, \ldots, \alpha_{N}\right) \succeq \alpha$ and $y=x \alpha_{1} A_{1} \ldots \alpha_{N} A_{N}$ where, for every $\alpha \in$ $\Pi_{c}, \bigcirc(\alpha)=\alpha$ and, for every $N \geq 1$ and for every $\alpha_{1}, \ldots, \alpha_{N} \in \Pi_{c}$, $\bigcirc\left(\alpha, \alpha_{1}, \ldots, \alpha_{N}\right)=\alpha ; \bigcirc\left(\alpha_{1}, \ldots, \alpha_{N}\right)$. A proof by induction on $M$ will show that:

Proposition 9 For every $M \geq 1$, for every $\alpha_{1}, \ldots, \alpha_{M} \in \Pi_{c}$, for every $N \geq 1$ and for every $\beta_{1}, \ldots, \beta_{N} \in \Pi_{c}, \bigcirc\left(\alpha_{1}, \ldots, \alpha_{M}\right) ; \bigcirc\left(\beta_{1}, \ldots, \beta_{N}\right)$ $\equiv \bigcirc\left(\alpha_{1}, \ldots, \alpha_{M}, \beta_{1}, \ldots, \beta_{N}\right)$.

Consequently, the reader may easily verify that :

Proposition $10(W, R)$ is a countable standard irreflexive frame on which $S$ is a function of maximality.

Consequently, $(W, R, S)$ is a countable standard irreflexive space of maximality called "space of subordination" and :

Theorem 2 For every $A \in \Phi_{c}$, if $A$ is true in every countable standard irreflexive space of maximality then $A$ is a theorem of $R A L$.

Proof If $A$ is not a theorem of $R A L$ then the singleton $\{\neg A\}$ is consistent and, according to the proposition 4, there exists a maximal and consistent set ?' of formulas such that $\neg A \in$ ?' $^{\prime}$. Let $(W, R, S)$ be a space of subordination such that $S(0)=$ ?'. Consequently, $A \notin$ $S(0), A$ is not true in $(W, R, S)$ and $A$ is not true in every countable 
standard irreflexive space of maximality.

The use of spaces of subordination have been introduced by Cresswell [12] and developed by Humberstone [13] and Balbiani [1, 2].

\section{Proof of the normal completeness}

Let $(W, R, S)$ be a countable standard irreflexive space of maximality. This section is devoted to the proof that $(W, R, S)$ can be transformed into a countable partial standard irreflexive space of maximality $\left(W_{\omega}, R_{\omega}, S_{\omega}\right)$ such that $W_{\omega} \supseteq W$ and $S_{\omega \mid W}=S$.

\section{$7.1 \quad$ Local completion}

Let $(W, R, S)$ be a countable standard irreflexive space of maximality, $\alpha, \beta \in \Pi_{c}$ and $x, z \in W$ be such that $x R(\alpha ; \beta) z . \quad(\alpha, \beta, x, z)$ is called "potential defect of $(W, R, S)$ ". Our assumptions immediately yield the result that $S(x)$ and $S(z)$ are maximal and consistent sets of formulas such that $[\alpha ; \beta] S(x) \subseteq S(z)$. Consequently, according to the proposition 6 , there exists a maximal and consistent set $\Delta$ of formulas such that $[\alpha] S(x) \subseteq \Delta$ and $[\beta] \Delta \subseteq S(z)$. Let $\left(W^{\prime}, R^{\prime}, S^{\prime}\right)$ be a space of subordination such that $S^{\prime}(0)=\Delta$. Let $W^{\prime \prime}=W \cup W^{\prime}, R^{\prime \prime}$ be the mapping of $\Pi_{c}$ to $2^{W^{\prime \prime} \times W^{\prime \prime}}$ such that, for every $\gamma \in \Pi_{c}$ and for every $t, u \in W^{\prime \prime}, t R^{\prime \prime}(\gamma) u$ iff :

- Either $t \in W, u \in W$ and $t R(\gamma) u$.

- Or $t \in W^{\prime}, u \in W^{\prime}$ and $t R^{\prime}(\gamma) u$.

- Or $t=x, u=0$ and $\alpha \succeq \gamma$.

- Or $t=x, u \in W^{\prime} \backslash\{0\}$ and there exists $\gamma^{\prime \prime} \in \Pi_{c}$ such that 0 $R^{\prime}\left(\gamma^{\prime \prime}\right) u$ and $\alpha ; \gamma^{\prime \prime} \succeq \gamma$.

- Or $t \in W \backslash\{x\}, u=0$ and there exists $\gamma^{\prime} \in \Pi_{c}$ such that $t R\left(\gamma^{\prime}\right)$ $x$ and $\gamma^{\prime} ; \alpha \succeq \gamma$.

- Or $t \in W \backslash\{x\}, u \in W^{\prime} \backslash\{0\}$ and there exists $\gamma^{\prime}, \gamma^{\prime \prime} \in \Pi_{c}$ such that $t R\left(\gamma^{\prime}\right) x, 0 R^{\prime}\left(\gamma^{\prime \prime}\right) u$ and $\gamma^{\prime} ;\left(\alpha ; \gamma^{\prime \prime}\right) \succeq \gamma$.

- Or $t=0, u=z$ and $\beta \succeq \gamma$.

- Or $t=0, u \in W \backslash\{z\}$ and there exists $\gamma^{\prime \prime} \in \Pi_{c}$ such that $z$ $R\left(\gamma^{\prime \prime}\right) u$ and $\beta ; \gamma^{\prime \prime} \succeq \gamma$. 
and $S^{\prime \prime}$ be the mapping of $W^{\prime \prime}$ to the set of the maximal and consistent sets of formulas such that, for every $t \in W, S^{\prime \prime}(t)=S(t)$ and, for every $t \in W^{\prime}, S^{\prime \prime}(t)=S^{\prime}(t)$. It is easy to verify that $\left(W^{\prime \prime}, R^{\prime \prime}\right)$ is a countable standard irreflexive frame on which $S^{\prime \prime}$ is a function of maximality. Consequently, $\left(W^{\prime \prime}, R^{\prime \prime}, S^{\prime \prime}\right)$ is a countable standard irreflexive space of maximality called "local completion of $(W, R, S)$ with respect to $\left(W^{\prime}, R^{\prime}, S^{\prime}\right)$ and $(\alpha, \beta, x, z)$ " such that $W^{\prime \prime} \supseteq W$ and $S_{\mid W}^{\prime \prime}=S$. Observe that there exists $y \in W^{\prime \prime}$ such that $x R^{\prime \prime}(\alpha) y$ and $y R^{\prime \prime}(\beta) z$.

\subsection{Global completion}

Let $(W, R, S)$ be a countable standard irreflexive space of maximality and $\mathcal{S}_{0}, \mathcal{S}_{1}, \mathcal{S}_{2}, \ldots$ be the sequence of countable standard irreflexive spaces of maximality defined by induction in the following way :

- Let $W_{0}=W, R_{0}=R, S_{0}=S$ and $\mathcal{S}_{0}=\left(W_{0}, R_{0}, S_{0}\right)$.

- For every $i \geq 1$, let $\left(\alpha_{1}, \beta_{1}, x_{1}, z_{1}\right),\left(\alpha_{2}, \beta_{2}, x_{2}, z_{2}\right), \ldots$ be a list of the potential defects of $\mathcal{S}_{i-1}$ and $\mathcal{S}_{i}^{0}, \mathcal{S}_{i}^{1}, \mathcal{S}_{i}^{2}, \ldots$ be the sequence of countable standard irreflexive spaces of maximality defined by induction in the following way :

- Let $W_{i}^{0}=W_{i-1}, R_{i}^{0}=R_{i-1}, S_{i}^{0}=S_{i-1}$ and $\mathcal{S}_{i}^{0}=\left(W_{i}^{0}, R_{i}^{0}, S_{i}^{0}\right)$.

- For every $j \geq 1$, let $\mathcal{S}_{i}^{j}=\left(W_{i}^{j}, R_{i}^{j}, S_{i}^{j}\right)$ be a local completion of $\mathcal{S}_{i}^{j-1}$ with respect to $\left(\alpha_{j}, \beta_{j}, x_{j}, z_{j}\right)$.

Then, let $W_{i}=\bigcup\left\{W_{i}^{j}: j \geq 0\right\}, R_{i}$ be the mapping of $\Pi_{c}$ to $2^{W_{i} \times W_{i}}$ such that, for every $\alpha \in \Pi_{c}$ and for every $x, y \in W_{i}, x$ $R_{i}(\alpha) y$ iff there exists $j \geq 0$ such that $x \in W_{i}^{j}, y \in W_{i}^{j}$ and $x R_{i}^{j}(\alpha) y, S_{i}$ be the mapping of $W_{i}$ to the set of the maximal and consistent sets of formulas such that, for every $x \in W_{i}$, there exists $j \geq 0$ such that $x \in W_{i}^{j}$ and $S_{i}(x)=S_{i}^{j}(x)$ and $\mathcal{S}_{i}$ $=\left(W_{i}, R_{i}, S_{i}\right)$. The reader may easily verify that $\left(W_{i}, R_{i}\right)$ is a countable standard irreflexive frame on which $S_{i}$ is a function of maximality. Consequently, $\mathcal{S}_{i}$ is a countable standard irreflexive space of maximality such that $W_{i} \supseteq W_{i-1}$ and $S_{i \mid W_{i-1}}=S_{i-1}$. Observe that, for every potential defect $(\alpha, \beta, x, z)$ of $\mathcal{S}_{i-1}$, there exists $y \in W_{i}$ such that $x R_{i}(\alpha) y$ and $y R_{i}(\beta) z$.

Then, let $W_{\omega}=\bigcup\left\{W_{i}: i \geq 0\right\}, R_{\omega}$ be the mapping of $\Pi_{c}$ to $2^{W_{\omega} \times W_{\omega}}$ such that, for every $\alpha \in \Pi_{c}$ and for every $x, y \in W_{\omega}, x R_{\omega}(\alpha) y$ iff there exists $i \geq 0$ such that $x \in W_{i}, y \in W_{i}$ and $x R_{i}(\alpha) y, S_{\omega}$ be 
the mapping of $W_{\omega}$ to the set of the maximal and consistent sets of formulas such that, for every $x \in W_{\omega}$, there exists $i \geq 0$ such that $x$ $\in W_{i}$ and $S_{\omega}(x)=S_{i}(x)$ and $\mathcal{S}_{\omega}=\left(W_{\omega}, R_{\omega}, S_{\omega}\right)$. It is easy to verify that $\left(W_{\omega}, R_{\omega}\right)$ is a countable standard irreflexive frame on which $S_{\omega}$ is a function of maximality. Consequently, $\mathcal{S}_{\omega}$ is a countable standard irreflexive space of maximality called "global completion of $(W, R, S)$ " such that $W_{\omega} \supseteq W$ and $S_{\omega \mid W}=S$. Observe that, for every potential defect $(\alpha, \beta, x, z)$ of $\mathcal{S}_{\omega}$, there exists $y \in W_{\omega}$ such that $x R_{\omega}(\alpha) y$ and $y$ $R_{\omega}(\beta) z$. Consequently, $\mathcal{S}_{\omega}$ is a countable partial standard irreflexive space of maximality and :

Theorem 3 For every $A \in \Phi_{c}$, if $A$ is true in every countable partial standard irreflexive space of maximality then $A$ is a theorem of $R A L$.

Proof If $A$ is not a theorem of $R A L$ then, according to the theorem 2, there exists a countable standard irreflexive space of maximality $(W, R, S)$ such that $A$ is not true in $(W, R, S)$. Let $\mathcal{S}_{\omega}$ be the global completion of $(W, R, S)$. Consequently, $A$ is not true in $\mathcal{S}_{\omega}$.

Clearly, we have demonstrated that :

Corollary 1 For every $A \in \Phi_{c}, A$ is a theorem of $R A L$ iff $A$ is true in every partial model iff $A$ is true in every normal model iff $A$ is true in every countable normal irreflexive model.

Proof This corollary is a consequence of the proposition 3, the theorem 1 , the proposition 8 and the theorem 3 .

\section{Conclusion}

To close our study of our relative modal logic with composition and intersection, we mention some questions that remain unsolved.

Consider the question of the complete axiomatization of a relative modal logic with composition, intersection and tests or a relative modal logic with composition, intersection and converse. Remark that, in the section 7.1, within the context of the local completion, the proof that $\left(W^{\prime \prime}, R^{\prime \prime}\right)$ is standard rests on the fact that the frames $(W, R)$ and $\left(W^{\prime}, R^{\prime}\right)$ are irreflexive.

Consider the issue of the complete axiomatization of the numerous relative modal logics with composition and intersection devised within 
the context of the research in deontic logic [14] and data analysis logic [15].

Add the composition operator of agents to the various logics of knowledge incorporating distributed knowledge $[4,5]$, the knowledge of the "compound agent" $\alpha ; \beta$ being the knowledge of the agent $\alpha$ about the knowledge of the agent $\beta$ and the knowledge of the "distributed agent" $\alpha \wedge \beta$ being the set of the conclusions to be drawn from the combined knowledge of the agents $\alpha$ and $\beta$ and consider the matter of the complete axiomatization of the resultant logics of knowledge.

Prove or disprove that $\succeq$ is decidable, that is to say $:$ is there an algorithm determining, for every $\alpha, \beta \in \Pi_{c}$, whether it is the case that $\alpha \succeq \beta$ ?

\section{Acknowledgement}

Special acknowledgement is heartly granted to Dimiter Vakarelov who made several helpful comments for improving the readability of the paper.

\section{References}

[1] P. Balbiani. Terminological modal logic. M. Kracht, M. de Rijke, H. Wansing, M. Zakharyaschev (editors), Advances in Modal Logic. Lecture Notes 87, 23-39, Center for the Study of Language and Information, 1998.

[2] P. Balbiani. Inequality without irreflexivity. Journal of Applied Non-Classical Logics, to appear.

[3] R. Danecki. Nondeterministic propositional dynamic logic with intersection is decidable. A. Skowron (editor), Computation Theory. Lecture Notes in Computer Science 208, 34-53, SpringerVerlag, 1985.

[4] R. Fagin, J. Halpern, Y. Moses, M. Vardi. Reasoning About Knowledge. MIT Press, 1995.

[5] R. Fagin, J. Halpern, M. Vardi. What can machines know ? On the properties of knowledge in distributed systems. Journal of the ACM, Volume 39, 328-376, 1992. 
[6] M. Fischer, R. Ladner. Propositional dynamic logic of regular programs. Journal of Computer and System Sciences, Volume 18, 194-211, 1979.

[7] R. Goldblatt. Axiomatising the Logic of Computer Programming. Lecture Notes in Computer Science 130, Springer-Verlag, 1982.

[8] R. Goldblatt. Logics of Time and Computation. Lecture Notes 7, Center for the Study of Language and Information, 1987.

[9] D. Harel. First-Order Dynamic Logic. Lecture Notes in Computer Science 68, Springer-Verlag, 1979.

[10] D. Harel. Recurring dominoes : making the highly undecidable highly understandable. M. Karpinski (editor), Foundations of Computation Theory. Lecture Notes in Computer Science 158, 177-194, Springer-Verlag, 1983.

[11] D. Harel. Dynamic logic. D. Gabbay, F. Guenthner (editors), Handbook of Philosophical Logic. Volume 3, 497-604, Reidel, 1984 .

[12] G. Hughes, M. Cresswell. A Companion to Modal Logic. Methuen, 1984.

[13] I. Humberstone. Inaccessible worlds. Notre Dame Journal of Formal Logic, Volume 24, 346-352, 1983.

[14] J.-J. Meyer. A different approach to deontic logic : deontic logic viewed as a variant of dynamic logic. Notre Dame Journal of Formal Logic, Volume 29, 109-136, 1988.

[15] E. Orłowska. Kripke models with relative accessibility and their applications to inferences from incomplete information. Banach Center Publications, Mathematical Problems in Computation Theory. Volume 21, 329-339, Polish Scientific Publishers, 1988.

[16] S. Passy, T. Tinchev. An essay in combinatory dynamic logic. Information and Control, Volume 93, 263-332, 1991. 